\title{
Polar Mesospheric Clouds (PMCs) Observed by the Ozone Monitoring Instrument (OMI) on Aura
}

\author{
Matthew T. DeLand ${ }^{1}$, Eric P. Shettle ${ }^{2}$, Pieternel F. Levelt ${ }^{3}$, and Matthew G. Kowalewski ${ }^{1,4}$ \\ ${ }^{\prime}$ Science Systems and Applications, Inc. (SSAI), 10210 Greenbelt Rd., Suite 600, Lanham, MD \\ 20706 \\ ${ }^{2}$ Remote Sensing Division, Code 7227, Naval Research Laboratory, Washington, DC $20375-$ \\ 5351 \\ ${ }^{3}$ Royal Netherlands Meteorological Institute (KNMI), NL-3730 AE De Bilt, Netherlands \\ ${ }^{4}$ Now at University of Maryland Baltimore County (UMBC), Baltimore, MD 21250
}

\begin{abstract}
Backscattered ultraviolet (BUV) instruments designed for measuring stratospheric ozone profiles have proven to be robust tools for observing polar mesospheric clouds (PMCs). These measurements are available for more than 30 years, and have been used to demonstrate the existence of long-term variations in PMC occurrence frequency and brightness. The Ozone Monitoring Instrument (OMI) on the EOS Aura satellite provides new and improved capabilities for PMC characterization. OMI uses smaller pixels than previous BUV instruments, which increases its ability to identify PMCs and discern more spatial structure, and its wide cross-track viewing swath provides full polar coverage up to $90^{\circ}$ latitude every day in both hemispheres. This cross-track coverage allows the evolution of PMC regions to be followed over several consecutive orbits. Localized PMC variations determined from OMI measurements are consistent with coincident SBUV/2 measurements. Nine seasons of PMC observations from $\mathrm{OMI}$ are now available, and clearly demonstrate the advantages of these measurements for PMC analysis.
\end{abstract}

\section{Introduction.}

Satellite measurements of backscattered ultraviolet (BUV) radiation have been used to monitor stratospheric profile ozone and total column ozone since the late 1960s [Heath et al., 1973]. Regular ozone observations began in November 1978 with the Solar Backscatter Ultraviolet (SBUV) and Total Ozone Mapping Spectrometer (TOMS) instruments on the Nimbus-7 satellite [Heath et al., 1975]. Additional TOMS instruments were flown on the Meteor-3 [Gleason et al., 1993], ADEOS [Krueger et al., 1998], and Earth Probe [McPeters et al., 1998] satellites through 2004. A second-generation version of the SBUV instrument, designated SBUV/2, was developed by NOAA for operational use. Seven SBUV/2 instruments have been flown since 1985 [e.g. Frederick et al., 1986; Hilsenrath et al., 1995; DeLand et al., 2001 ], and four SBUV/2 instruments are currently operating.

Polar mesospheric clouds (PMCs) are tenuous, narrow layers of ice crystals that form at 80-85 km altitude only during a well-defined season in each hemisphere (approximately 20-30 days before summer solstice to 60-70 days after solstice) [e.g. Thomas, 1995]. PMCs are generally observed at latitudes poleward of $\sim 50^{\circ}$, although observations down to $40^{\circ} \mathrm{N}$ do occur [e.g. Taylor et al., 2002]. Thomas et al. [1991] demonstrated that Nimbus-7 SBUV 
measurements of backscattered albedo, at wavelengths between $252 \mathrm{~nm}$ and $293 \mathrm{~nm}$, could be analyzed to identify the presence and brightness of PMCs in the upper atmosphere. Since these measurements are taken during the normal operating mode of the SBUV instrument, PMC information can be derived from data obtained by all subsequent SBUV/2 instruments as well. PMC results from combined SBUV and SBUV/2 data sets have been presented in numerous papers [e.g. DeLand et al., 2003; Thomas et al., 2005; DeLand et al., 2007; Shettle et al., 2009]. These results have demonstrated the existence of two significant long-term variations in PMC occurrence frequency and brightness: Increasing trends at most latitudes in both hemispheres, and an anti-correlation with solar activity over solar cycle time scales. However, our ability to evaluate SBUV/2 measurements for PMC properties and morphology is constrained by the nadir viewing geometry and large field of view (nominal size $=11.3^{\circ} \times 11.3^{\circ}$ $=170 \mathrm{~km} \times 170 \mathrm{~km}$ at the Earth's surface). The SBUV/2 instrument samples wavelengths sequentially with a 2 -second interval during normal operations, so that the field of view moves an additional $70 \mathrm{~km}$ along the orbit track during the measurement of the five wavelengths used in PMC analysis. The complex small-scale PMC structures shown by data from the Cloud Imaging and Particle Size (CIPS) instrument on the Aeronomy of Ice in the Mesosphere (AIM) satellite [Rusch et al., 2009] clearly shows that SBUV/2 measurements typically observe both PMC and non-PMC regions in every sample. This averaging process thus reduces the ability of SBUV/2 measurements to detect faint PMCs, as well as narrow and isolated bands of bright PMCs.

The Ozone Monitoring Instrument (OMI) is a hyperspectral instrument flying on the EOS Aura satellite to continue the stratospheric profile ozone and total column ozone record [Levelt et al., 2006a]. Aura was launched on July 15, 2004, and regular operations began in December 2004. OMI measurements have a smaller pixel size and wider geographic coverage than SBUV/2 measurements, offering significant advantages for PMC detection and analysis. Approximately 30-40 OMI nadir view pixels fall within a single SBUV/2 footprint, improving the ability to identify small-scale PMC structure. OMI observes most locations at typical PMC latitudes $\left(>55^{\circ}\right)$ on two or more consecutive orbits each day, and its coverage extends to $90^{\circ}$ latitude in both hemispheres. This paper presents an overview of the OMI instrument and the adaptation of the SBUV/2 PMC detection algorithm for use with OMI data.

\section{OMI Instrument and Data Description.}

Detailed descriptions of the OMI instrument design and operation are provided by Levelt et al. [2006b] and Dobber et al. [2006]. OMI is a single monochromator that covers the wavelength range 264-504 nm, separated into three spectral ranges with overlapping coverage. The measurements used for PMC analysis are taken from the UV1 channel, which covers the region between $264-311 \mathrm{~nm}$ with $0.63 \mathrm{~nm}$ spectral resolution. OMI views the Earth in the nadir direction using a wide field-of-view (FOV) telescope with a total cross-track coverage of $\sim 114^{\circ}$ (Figure 1). The UV1 data are binned into 30 rows with equal angular extent, yielding pixel cross-track widths of $48 \mathrm{~km}$ at nadir (rows \#15-16), increasing to $\sim 200 \mathrm{~km}$ at the outer edges (rows \#1 and \#30). OMI co-adds five consecutive measurements with an exposure time of 0.4 seconds per measurement, which gives a nominal pixel size of $13 \mathrm{~km}$ (along-track) x $48 \mathrm{~km}$ (cross-track) in the nadir rows, increasing to $\sim 25 \mathrm{~km} \mathrm{x} 200 \mathrm{~km}$ at the outer edges of the viewing range (Figure 2). We do not use rows \#1 and \#30 in this analysis due to problems with signal-tonoise performance. Over the course of OMI's 14 daily orbits, the total cross-track swath of 
approximately $2600 \mathrm{~km}$ provides nearly full global coverage at the Equator, with substantial overlap between consecutive orbits at PMC latitudes.

Instrument throughput changes in the UV1 spectral region have been analyzed using polar ice cap radiances [Jaross and Warner, 2008]. The OMI calibration has been shown to be stable to better than $0.5 \%$ [G. Jaross, private communication]. Since OMI is a single monochromator, the radiance measurements at wavelengths less than $300 \mathrm{~nm}$ can have significant stray light contributions from longer wavelengths because of the rapid spectral variation in backscattered radiance [e.g. DeLand et al., 2001]. The operational OMI data employ a stray light correction algorithm developed from both prelaunch calibration tests and inflight measurements using dedicated rows of the CCD. Dobber et al. [2008] describes the use of both spatial and spectral stray light corrections in the creation of OMI Level $1 \mathrm{~b}$ data. These corrections are estimated to reduce the stray light contamination for UV1 measurements to less than $5 \%$.

We use OMI Level $1 \mathrm{~b}$ radiance data from the Collection 3 processing in this analysis. These data include quality flags for a variety of parameters at the pixel, swath, daily, and global levels of instrument data [van den Oord et al., 2006]. A specific flag of interest is the random telegraph signal (RTS) flag, which indicates raw pixels whose dark current signal tends to shift between multiple stable levels as a result of proton impact damage [Dobber et al., 2006]. Approximately 30-50 raw pixels are averaged into each albedo bin used for PMC analysis. Detailed examination does not currently show an impact on OMI PMC retrievals when RTS flags are present in the $1 \mathrm{~b}$ data. However, we do save these flag values in our PMC data sets, and we will continue to track the behavior of the retrievals.

OMI Level 2 total ozone data began to show row-dependent anomalies in June 2007. These anomalies were soon identified in Level $1 \mathrm{~b}$ UV1 radiance data as well, and have continued to evolve with time. The detailed behavior of the row anomaly is complex, but the general effect is to enhance observed radiance signals in affected rows in the Northern Hemisphere PMC region (latitude $>40^{\circ}$ ) by factors up to $5-10 x$, and to decrease observed Southern Hemisphere signals in affected rows by factors of $0.6-0.8$. The magnitude of the observed effect also varies with solar zenith angle (SZA). Detailed discussions of the row anomaly behavior can be found at http:/www.knminlomi/research/product/rowanomaly-background.php. At this time, no correction process is available for these effects in UV1 data. Based on inspection of PMC analysis results, we have chosen to omit any row from our data processing if its background deviation from the SZA-dependent variation of the unaffected rows is greater than $10 \%$. This represents rows \#16-23 for the NH 2008 season, and rows \#14-23 for the SH 2008-2009 season. Initial NH 2009 results show an expansion of the contaminated region in UV1 data to cover rows \#7-26. We will continue to track the status of instrument throughput changes and row anomaly behavior.

\section{OMI PMC Detection Algorithm.}

The measurement technique and spectral coverage of OMI allow us to adapt the SBUV PMC detection algorithm [Thomas et al., 1991; DeLand et al., 2003, 2007] to analyze OMI data. This algorithm looks for pixels with albedo enhancements relative to the Rayleigh scattering 
background using data at the five shortest SBUV wavelengths $(252-292 \mathrm{~nm})$, and uses a combination of four tests to distinguish pixels containing PMCs. Since OMI spectra have a slightly different wavelength scale for each swath, we interpolate all spectra to a regular grid with $\Delta \lambda=0.5 \mathrm{~nm}$ for consistent processing. OMI spectral coverage does not extend as low as SBUV/2 instruments, so we adopted the revised wavelength set 267.0, 275.0, 283.5, 287.5, 292.5 $\mathrm{nm}$ for OMI use. We plan to conduct further studies to investigate possible benefits of using different (or expanded) wavelength sets. We found that averaging albedo values for three consecutive $0.5 \mathrm{~nm}$ bins at each PMC analysis wavelength significantly improved the noise in the albedo data.

The Rayleigh scattering background atmospheric albedo observed by OMI at the wavelengths used for PMC analysis varies by almost an order of magnitude with changing solar zenith angle, and the off-nadir measurements typically show increased albedo values relative to nadir measurements by up to a factor of two due to the increased viewing path through the atmosphere. In order to allow processing of all OMI data with a single set of algorithm parameters, we scale the data for each row to be consistent with nadir-viewing measurements using geometric terms which are independent of the variable atmospheric properties. The following discussion presents the derivation of this scaling procedure.

The background atmospheric albedo, $A_{\text {Ray }}$, due to molecular Rayleigh scattering in the presence of ozone absorption is given by Bailey et al. [2009], who generalized an earlier result of McPeters [1980]:

$$
A_{\text {Ray }}=P_{\text {Ray }}(\Theta) \Gamma(\sigma+1) \beta N\left(\mathrm{z}_{0}\right) /\left\{\mu_{\mathrm{V}}\left[\left(1 / \mu_{\mathrm{V}}\right)+C h\left(\mu_{\mathrm{SZA}}, \mathrm{H}_{\mathrm{oz}}\right)\right]^{\sigma} \alpha^{\sigma} C\left(\mathrm{z}_{0}\right)^{\sigma}\right\}
$$

$P_{\text {Ray }}(\Theta)$ is the Rayleigh scattering phase function for a scattering angle $\Theta, \Gamma$ is the gamma function, $\sigma$ is the ratio of the ozone scale height $\left(\mathrm{H}_{\mathrm{oz}}\right)$ to the atmospheric scale height (assumed to be constant for the altitudes of interest), $\beta$ is the cross section for Rayleigh scattering, $N$ is the column density of atmospheric molecules above a reference altitude $z_{0}, \mu_{V}$ is the cosine of the view zenith angle, $\mu_{S Z A}$ is the cosine of the solar zenith angle, $C h$ is the Chapman function [Chapman, 1931], $\alpha$ is the cross section for pure absorption by ozone, and $C$ is the column density of ozone molecules above that reference altitude. For solar zenith angles less than $70^{\circ}$ the Chapman function reduces to $1 / \mu_{S Z A}$.

Equation [1] for the atmospheric albedo can be separated into two factors, $F\left(\mathrm{z}_{0}\right)$ which just depends on the atmospheric properties and $G\left(\mu_{\mathrm{V}}, \mu_{\mathrm{SZA}}\right)$ which depends primarily on the viewing/sun geometry. These factors are defined as:

$$
F\left(\mathrm{z}_{0}\right)=\Gamma(\sigma+1) \beta N\left(\mathrm{z}_{0}\right) /\left[\alpha^{\sigma} C\left(\mathrm{z}_{0}\right)^{\sigma}\right]
$$

and

$$
G\left(\mu_{\mathrm{V}}, \mu_{\mathrm{SZA}}\right)=P_{\mathrm{Ray}}(\Theta) /\left\{\mu_{\mathrm{V}}\left[\left(1 / \mu_{\mathrm{V}}\right)+C h\left(\mu_{\mathrm{SZA}}, \mathrm{H}_{\mathrm{oz}}\right)\right]^{\sigma}\right\}
$$

It will be noted that the geometric factor, $G$, still has some dependence on the atmospheric properties through the ozone scale height, $\mathrm{H}_{\mathrm{oz}}$, and exponent $\sigma$, the ratio of the ozone scale 
height to the atmospheric scale height. However, for the wavelengths of interest (267 to 292.5 $\mathrm{nm}$ ), ozone absorption limits the backscattered radiance viewed by the satellite to altitudes between approximately $40-60 \mathrm{~km}$. At these altitudes, the typical values of $\sigma$ vary from 0.5 to 0.65 , and the use of a median value between 0.55 and 0.58 in Equation [3] reproduces the albedo variation with solar zenith angle to $10-20 \%$. These variations are due primarily to uncertainties in $\sigma$, which is taken to be 0.58 , rather than uncertainties in $\mathrm{H}_{\mathrm{oz}}$ affecting the value of the Chapman function. Even a factor of two change in the ozone scale height changes the geometric factor, G, by as much as $10 \%$ only for our most extreme solar zenith angle of $88^{\circ}$ Dividing the observed atmospheric albedo $A_{\mathrm{obs}}$ by the geometric factor $G$ given in Equation [3] then removes most of the variation of the atmospheric albedo due to changes in the scattering geometry.

$$
A_{\text {scaled }}=A_{\text {obs }} / G\left(\mu_{\mathrm{V}}, \mu_{\mathrm{SZA}}\right)
$$

This makes it easier to approximate and remove the background atmospheric radiance from the measurements with the clouds. Using this procedure, we find cross-track agreement in absolute albedo between rows of OMI data to better than $10 \%$, and gives adjusted albedo values for each row in a format and magnitude consistent with SBUV/2 data. The agreement in albedo between rows reduces the uncertainty of the background albedo fit to $1-2 \%$.

For SBUV/2 data, measurements from all orbits for a single day are combined before calculating the background fit for PMC analysis. The higher along-track sampling rate of OMI compared to SBUV/2 $(13 \mathrm{~km} v s . \sim 220 \mathrm{~km})$ provides sufficient measurement density to process each OMI row separately, and furthermore allows each OMI row to be analyzed individually within a given orbit. This allows the background fit to the adjusted albedo to be more responsive to any longitudinal variations in stratospheric ozone. We used out-of-season measurements to represent typical atmospheric variability, and calculated the albedo standard deviation as a function of latitude at each wavelength. A quadratic fit to these data provides a threshold function that is used to screen out anomalous samples, and is also used to define the residual albedo threshold at $267 \mathrm{~nm}$ for PMC detection (Figure 3). Note that the relationship between latitude and solar zenith angle varies during the PMC season. We found that scaling this threshold function up by a factor of 1.6 was required to decrease PMC occurrence frequencies below $1 \%$ at the start and end of our nominal season ( 30 days before summer solstice to 70 days after solstice) [DeLand et al., 2003], as expected based on results from more sensitive satellite instruments such as SNOE [Bailey et al., 2005]. The combination of higher spatial resolution and greater geographic coverage means that OMI detects many more PMCs than an SBUV/2 instrument, which greatly improves our statistical basis for examining PMC behavior.

\section{Sample Results.}

Figure 4 shows an example of the OMI PMC albedo values retrieved for orbit 15878 on July 10, 2007 (day 191). Coherent structure is visible between $70^{\circ}-80^{\circ}$ latitude in numerous places, including a large bright region at $68^{\circ}-73^{\circ} \mathrm{N}$ and $75^{\circ}-95^{\circ} \mathrm{W}$. Figure 5 shows the residual albedo for row $\# 17$ only, which extends from approximately $40^{\circ} \mathrm{N}, 50^{\circ} \mathrm{W}$ to $65^{\circ} \mathrm{N}, 170^{\circ} \mathrm{E}$ in Figure 4. Structure at the sub- $100 \mathrm{~km}$ scale is evident at SZA $=70^{\circ}-80^{\circ}$. Note that some pixels with residual albedo values exceeding the threshold shown in Figure 3 are not classified as PMCs based on the results from additional detection tests, as described in DeLand et al. [2003, 
2007]. In some locations of this orbit, retrieved cloud albedo values can exceed $50 \times 10^{-6} \mathrm{sr}^{-1}$. This is brighter than almost any PMC albedo value observed by an SBUV/2 instrument, and is a natural consequence of the fact that it is easier to fill a $750 \mathrm{~km}^{2}$ OMI pixel with PMCs than a $22,500 \mathrm{~km}^{2}$ SBUV/2 pixel. OMI off-nadir measurements can have significantly different scattering angles (up to 20-30 degrees higher or lower) than the coincident sub-satellite measurements, so that ice phase function variations may affect the observed PMC brightness [Bailey et al., 2009]. Some of the residual albedo values are negative because of the remaining uncertainties in our fit of the background, which is subtracted from measurements, as may be noted in Figure 5.

The orbits of the Aura (with OMI) and NOAA-18 (with SBUV/2) satellites have very similar Equator-crossing times at different altitudes (and therefore have different orbital periods), so that Aura passes beneath NOAA-18 every 31 orbits, providing good spatial and temporal $(\Delta t$ $<15$ minutes) coincidences [Shettle et al., SBUV observations of polar mesospheric clouds compared with MLS temperatures and water vapor measurement, in preparation]. For these situations, we find that selecting OMI pixels whose center lies within $75 \mathrm{~km}$ of the center of the SBUV/2 field of view (FOV) gives approximately 25-28 OMI pixels within each SBUV/2 FOV. Figure 6 shows a comparison of the NOAA-18 SBUV/2 residual albedo values at $252 \mathrm{~nm}$ for 28 SBUV/2 events on July 3,2007 with the average of all coincident OMI residual albedo values at $267 \mathrm{~nm}$, inside the FOV for each SBUV/2 measurement. Squares indicate the samples identified as PMCs by the SBUV/2 algorithm. The correspondence is reasonably linear despite the relatively small number of data points, with a correlation coefficient of 0.87 . The majority of the PMC detections have higher albedo values for OMI than for SBUV/2, consistent with our previous statement that $\mathrm{OMI}$ is capable of identifying brighter clouds.

The large geographic coverage of OMI measurements makes it possible to track bright PMC regions over successive orbits. Figure 7 shows the retrieved PMC albedo for six consecutive orbits on 2007 day 191 (orbits 15873-15878), focusing on the region $60^{\circ}-90^{\circ} \mathrm{N}$. A bright region is visible to the west of Greenland at $\sim 70^{\circ} \mathrm{N}$ on orbit 15874 , and moves to the west on subsequent orbits. The brightness and internal structure of the region also evolves during this time. NOAA-17 and NOAA-18 SBUV/2 observations on this date also show the presence of a bright region of PMCs near Greenland. The orbit-by-orbit OMI observations also indicate the presence of extended voids at high latitudes $\left(\right.$ e.g. $80^{\circ}-90^{\circ} \mathrm{N}$ and $110^{\circ}-140^{\circ} \mathrm{W}$ in orbit 15878$)$, as first noted by Rusch et al. [2009].

Figure 8 shows the seasonal variation of PMC occurrence frequency observed by OMI in $5^{\circ}$ zonal average latitude bands for the $\mathrm{NH} 2007$ season. Relatively few PMCs are seen below $\sim 60^{\circ} \mathrm{N}$. Daily average occurrence frequency values of $40-50 \%$ are commonly seen above $80^{\circ} \mathrm{N}$, again higher than corresponding values for SBUV/2 instruments. Figure 9 shows the corresponding seasonal frequency plot for the $\mathrm{SH}$ 2007-2008 season. PMC frequency values are lower than $\mathrm{NH}$ values for latitudes less than $70^{\circ}$, but become significantly higher above $80^{\circ}$ latitude. It should be noted that typical scattering angle values at $85^{\circ} \mathrm{S}$ range between $80^{\circ}-90^{\circ}$, whereas scattering angle values at $85^{\circ} \mathrm{N}$ are approximately $110^{\circ}-120^{\circ}$. The ice phase function tends to be smaller at larger scattering angles, although the magnitude of the difference depends on details of the corresponding particle size distribution. Thus, increased PMC albedo values due to ice phase function effects may lead to higher observed occurrence frequency values in the 
Southern Hemisphere high latitude data. A complete interpretation of the PMC latitude dependence as observed by OMI also requires an understanding of the PMC local time variation, which is discussed further in DeLand et al. [2010].

\section{Conclusions.}

The OMI instrument represents an important advancement in satellite observations of PMCs. While its basic design provides continuity with the multi-decade SBUV series of instruments, the optical design and detector characteristics of OMI greatly improve its usefulness for PMC analysis. The use of a 2-D CCD detector produces smaller spatial pixels with continuous UV spectra for every pixel. The wide cross-track measurement swath provides daily observations up to $90^{\circ}$ latitude in each hemisphere, and gives multiple coincident samples every day at most PMC latitudes. DeLand et al. [2010] describes how we utilize this expanded temporal sampling to characterize PMC local time variations. In the future, we intend to use the spectral coverage of the OMI measurements to derive Angstrom exponent values and thus investigate PMC particle size behavior.

\section{Acknowledgements}

Most of this work was supported by grant NNH08CD48C from NASA's Office of Earth Science. Part of E. P. Shettle's work was supported by NRL internal funding from the Office of Naval Research. We greatly appreciate many insightful discussions with Gary Thomas and John Olivero. We acknowledge the valuable comments from three anonymous reviewers. 


\section{References}

Bailey, S. M., A. W. Merkel, G. E. Thomas, and J. N. Carstens (2005), Observations of polar mesospheric clouds by the Student Nitric Oxide Explorer, J. Geophys. Res., 110, D13203, doi:10.1029/2004JD005422.

Bailey, S. M., G. E. Thomas, D. W. Rusch, A. W. Merkel, C. D. Jeppesen, J. N. Carstens, C. E. Randall, W. E. McClintock, and J. M. Russell III (2009), Phase functions of polar mesospheric cloud ice as observed by the CIPS instrument on the AIM satellite, J. Atmos. Solar-Terr. Phys., 71, 373-380.

Bhartia, P. K., R. D. McPeters, C. L.,Mateer, L. E. Flynn, and C. Wellemeyer (1996), Algorithm for the estimation of vertical ozone profile from the backscattered ultraviolet (BUV) technique, J. Geophys. Res., 101, 18793-18806.

Bodhaine, B. A., N. B. Wood, E. G. Dutton, and J. R. Slusser (1999), On Rayleigh optical depth calculations, J. Atmos. Ocean. Tech., 16, 1854-1861.

Chapman, S. (1931), The absorption and dissociative or ionizing effect of monochromatic radiation in an atmosphere on a rotating earth, Part II. Grazing incidence, Proc. Phys. Soc. (London), 43, 483-501.

DeLand, M. T., R. P. Cebula, L.-K. Huang, S. L. Taylor, R. S. Stolarski, and R. D. McPeters (2001), Observations of "hysteresis" in backscattered ultraviolet ozone data, J. Atmos. Oceanic Tech., 18, 914-924.

DeLand, M. T., E. P. Shettle, G. E. Thomas, and J. J. Olivero (2003), Solar backscattered ultraviolet (SBUV) observations of polar mesospheric clouds (PMCs) over two solar cycles, J. Geophys. Res., I08(D8), 8445, doi:10.1029/2002JD002398.

DeLand, M. T., E. P. Shettle, G. E. Thomas, and J. J. Olivero (2007), Latitude-dependent longterm variations in polar mesospheric clouds from SBUV version $3 \mathrm{PMC}$ data, J. Geophys. Res., 112, D10315, doi:10.1029/2006JD007857.

DeLand, M. T., E. P. Shettle, G. E. Thomas, and J. J. Olivero (2010), Direct observations of PMC local time variations by Aura OMI, submitted to J. Atmos. Solar-Terr. Phys.

Dobber, M., et al. (2006), Ozone Monitoring Instrument calibration, IEEE Transactions on Geoscience and Remote Sensing, 44, 1209-1238.

Dobber, M., Q. Kleipool, R. Dirksen, P. Levelt, G. Jaross, S. Taylor, T. Kelly, L. Flynn, G. Leppelmeier, and N. Rozemeijer (2008), Validation of Ozone Monitoring Instrument level 1b data products, J. Geophys. Res., 113, D15S06, doi:10.1029/2007JD008665.

Frederick, J. E., R. P. Cebula, and D. F. Heath (1986), Instrument characterization for the detection of long-term changes in stratospheric ozone: An analysis of the SBUV/2 
radiometer, J. Atmos. Oceanic Technol., 3, 472-480.

Gleason, J. F., et al. (1993), Record low global ozone in 1992, Science, 260, 523-526.

Heath, D. F., C. L. Mateer, and A. J. Krueger (1973), The Nimbus-4 Backscattered Ultraviolet (BUV) atmospheric ozone experiment - Two years' operation, Pure Appl. Geophys., 106$108,1238-1253$.

Heath, D. F., A. J. Krueger, H. A. Roeder, and B. D. Henderson (1975), The Solar Backscatter Ultraviolet and Total Ozone Mapping Spectrometer (SBUV/TOMS) for Nimbus G, Opt. Eng., 14, 323-331.

Hilsenrath, E., R. P. Cebula, M. T. DeLand, K. Laamann, S. Taylor, C. Wellemeyer, and P. K. Bhartia (1995), Calibration of the NOAA-11 solar backscatter ultraviolet (SBUV/2) ozone data set from 1989 to 1993 using in-flight calibration data and SSBUV, $J$. Geophys. Res., 100, 1351-1366.

Jaross, G., and J. Warner (2008), Use of Antarctica for validating reflected solar radiation measured by satellite sensors, J. Geophys. Res., 113, D16S34, doi:10/1029/2007JD008835.

Krueger, A. J., et al. (1998), ADEOS Total Ozone Mapping Spectrometer (TOMS) Data Product User's Guide, NASA/TP-98-206857, 62 pp.

Levelt, P. F., E. Hilsenrath, G. W. Leppelmeier, G. H. J. van den Oord, P. K. Bhartia, J. Tamminen, J. F. de Haan, and J. P. Veefkind (2006a), Science objectives of the Ozone Monitoring Instrument, IEEE Transactions on Geoscience and Remote Sensing, 44, 1199-1208.

Levelt, P. F., G. H. J. van den Oord, M. R. Dobber, A. Mälkki, H. Visser, J. de Vries, P. Stammes, J. O. V. Lundell, and H. Saari (2006b), The Ozone Monitoring Instrument, IEEE Transactions on Geoscience and Remote Sensing, 44, 1093-1101.

McPeters, R. D. (1980), The behavior of ozone near the stratopause from two years of BUV observations, J. Geophys. Res., 85, 4545-4550.

McPeters, R., et al. (1998), Earth Probe Total Ozone Mapping Spectrometer (TOMS) Data Product User's Guide, NASA/TP-1998-206895, 70 pp.

Rusch, D. W., G. E. Thomas, W. McClintock, A. W. Merkel, S. M. Bailey, J. M. Russell III, C. E. Randall, C. Jeppesen, and M. Callan (2009), The cloud imaging and particle size instrument on the aeronomy of ice in the mesosphere mission: Cloud morphology for the northern 2007 season, J. Atmos. Solar-Terr. Phys., 71, 356-364.

Shettle, E. P., M. T. DeLand, G. E. Thomas, and J. J. Olivero (2009), Long term variations in the frequency of polar mesospheric clouds in the Northern Hemisphere from SBUV, 
Geophys. Res. Lett., 36, L02803, doi:10.1029/2008GL036048.

Taylor, M. J., M. Gadsden, R. P. Lowe, M. S. Zalcik, and J. Brausch (2002), Mesospheric cloud observations at unusually low latitudes, J. Atmos. Solar-Terr. Phys., 64, 991-999.

Thomas, G. E. (1995), Climatology of polar mesospheric clouds: Interannual variability and implications for long-term trends, in The Upper Mesosphere and Lower Thermosphere: A Review of Experiment and Theory, vol. 87, edited by R. M. Johnson and T. L. Killeen, pp. 185-200, AGU, Washington, DC.

Thomas, G. E., J. J. Olivero, M. DeLand, and E. P. Shettle (2003), Comment on "Are noctilucent clouds truly a 'miner's canary' for global change?", Eos Trans. AGU, 84, 352-353.

Thomas, G. E., R. D. McPeters, and E. J. Jensen (1991), Satellite observations of polar mesospheric clouds by the Solar Backscattered Ultraviolet radiometer: Evidence of a solar cycle dependence, J. Geophys. Res., 96, 927-939.

van den Oord, G. H. J., J. P. Veefkind, P. F. Levelt, and M. R. Dobber (2006), OMI level 0 to 1 b processing and operational aspects, IEEE Transactions on Geoscience and Remote Sensing, 44, 1380-1397. 


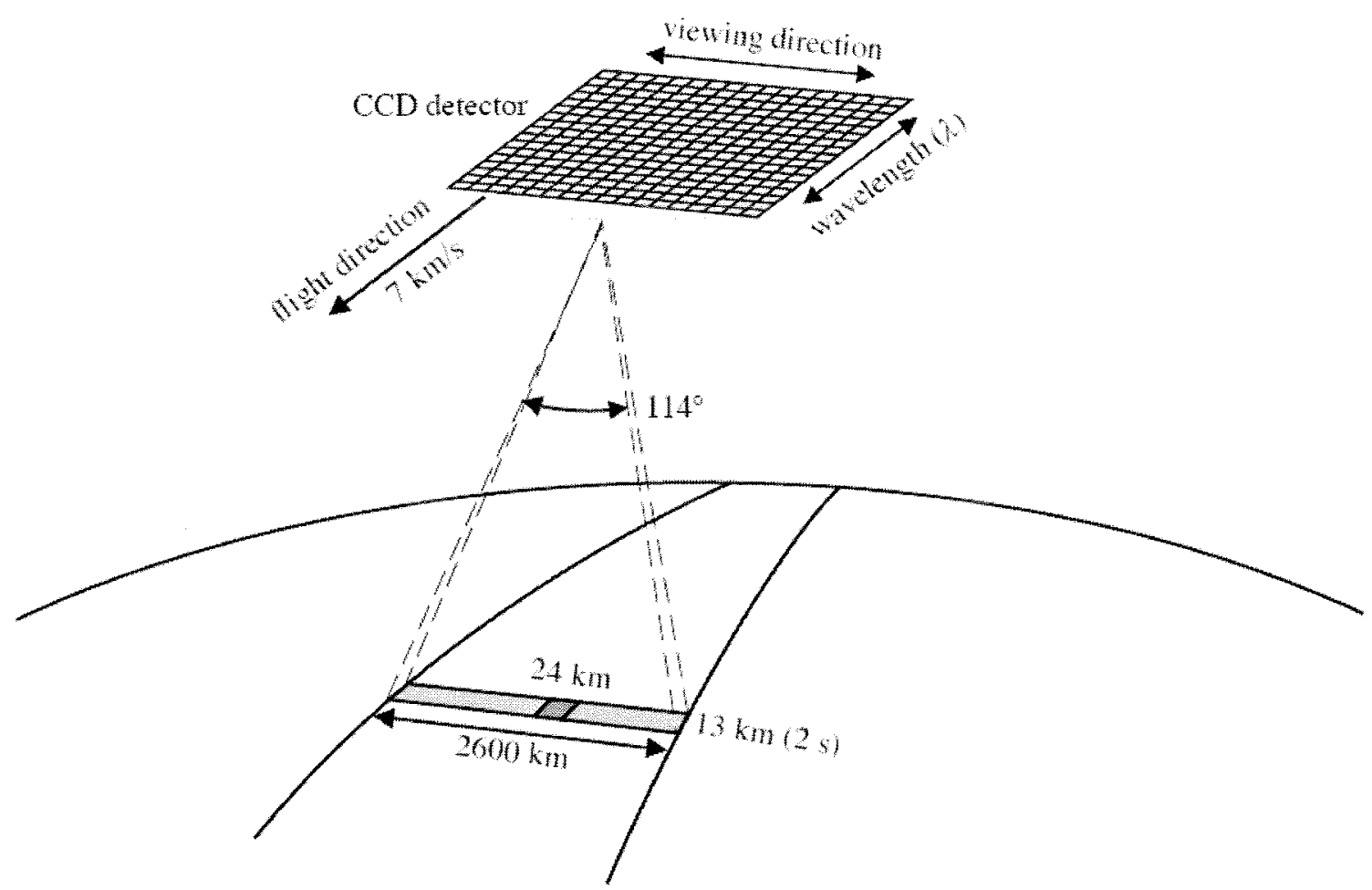

Figure 1. Diagram of OMI measurement sequence. Figure provided by Dutch Space and adapted from Levelt et al. [2006b]. 


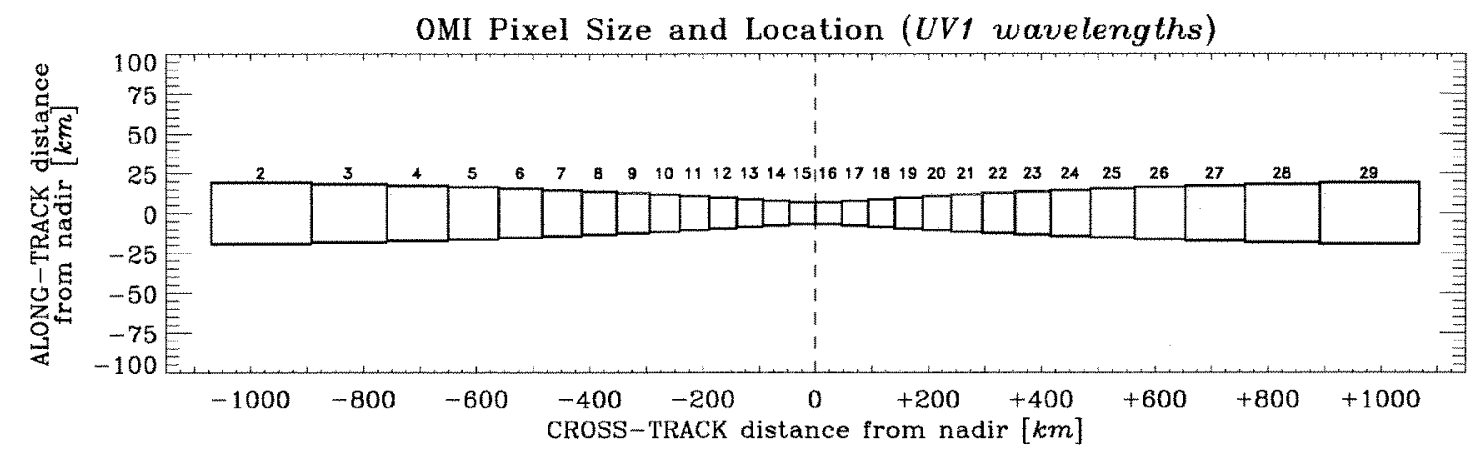

Figure 2. OMI pixel size and row location for UV1 wavelengths relative to orbit track. Note that the cross-track and along-track plot scales are different. 
OMI PMC Detection Threshold

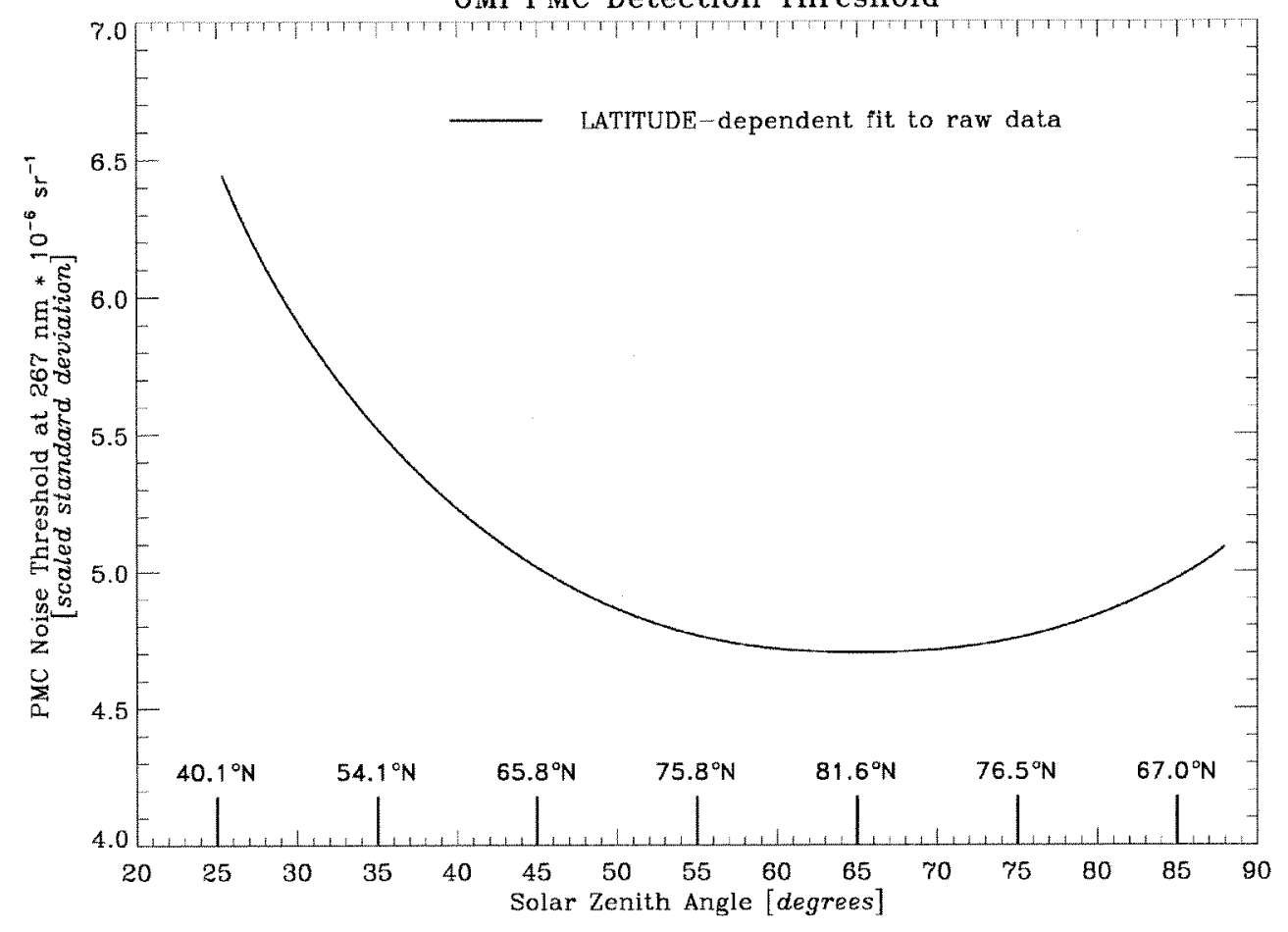

Figure 3. Albedo threshold at $267 \mathrm{~nm}$ for PMC detection in the OMI algorithm. The large tick marks indicate the Northern Hemisphere latitude values in row \#15 corresponding to specific solar zenith angle values on May 1 . 


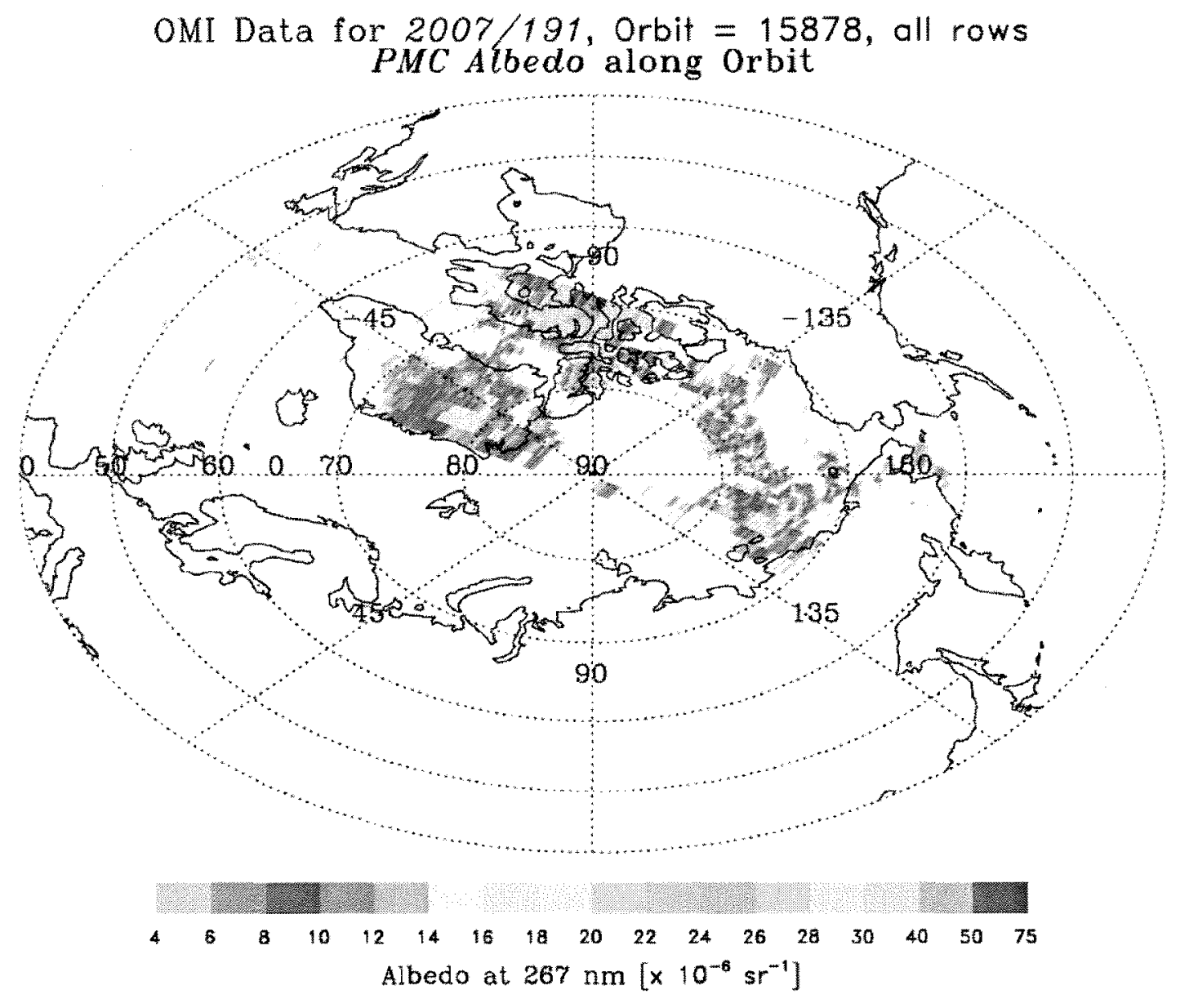

Figure 4. OMI PMC albedo values at $267 \mathrm{~nm}$ for all detections during orbit 15878 on July 10 , 2007. Data are shown for $50^{\circ}-90^{\circ} \mathrm{N}$ latitude. 


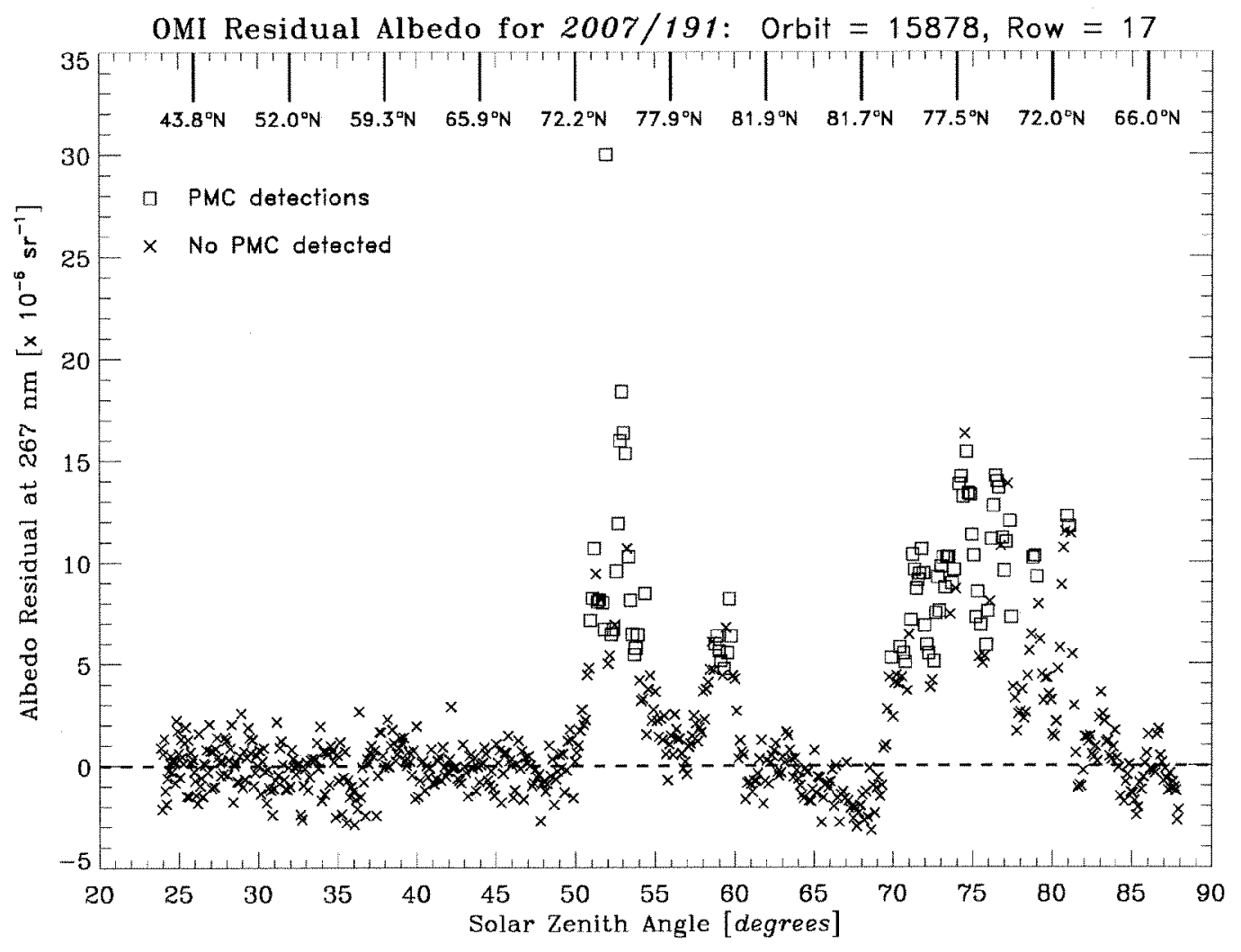

Figure 5. OMI albedo residual values [observed - background] at $267 \mathrm{~nm}$ for all samples from row \#17 of orbit 15878 on July 10,2007, plotted as a function of solar zenith angle. Squares represent pixels where a PMC was detected, and crosses represent no PMC detection. The corresponding latitudes for regularly spaced locations are identified with tick marks at the top of the plot. 


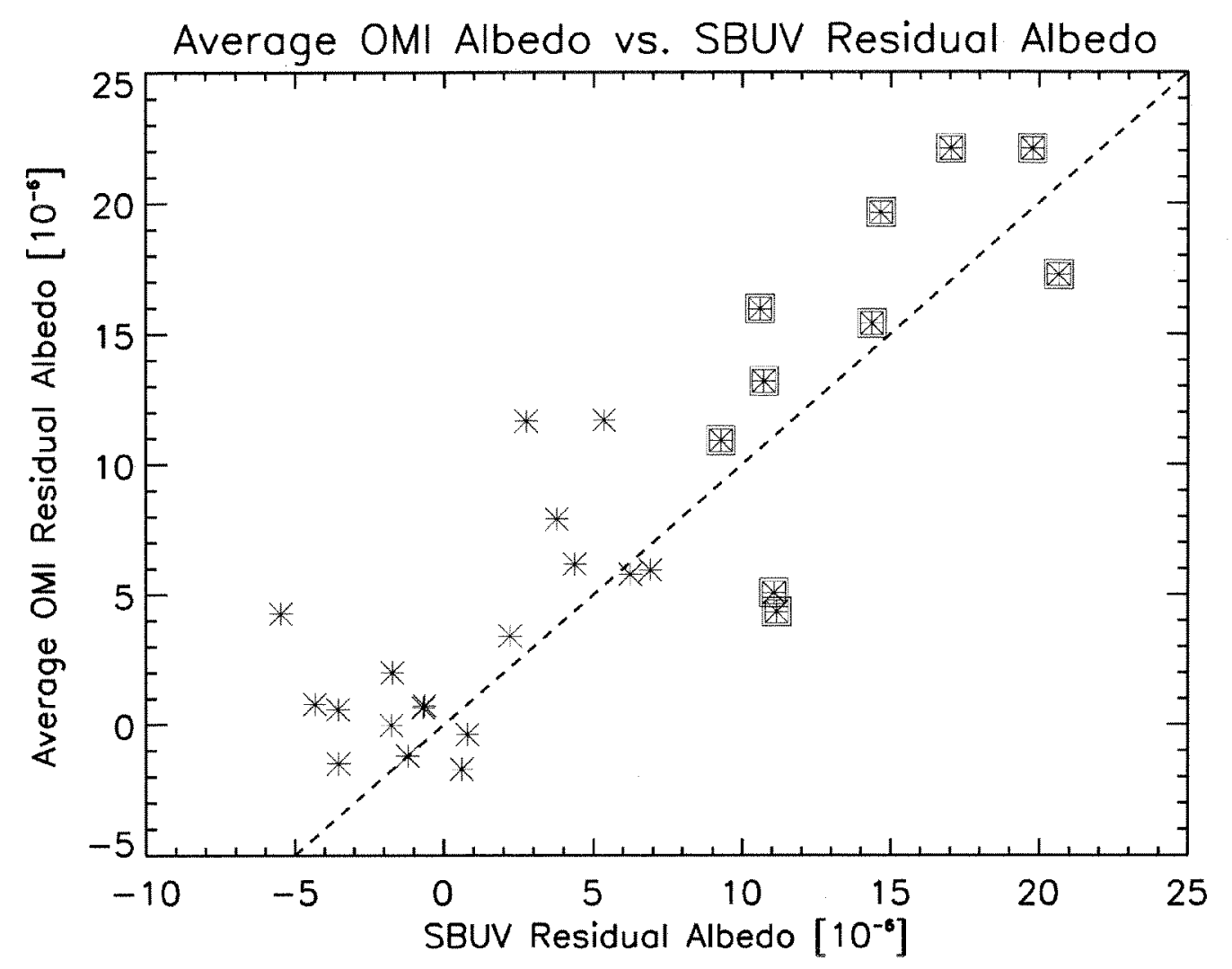

Figure 6. Residual albedo values for coincident NOAA-18 SBUV/2 and OMI measurements on July 3,2007. Coincidence criteria are defined in the text. Squares identify the samples where a PMC was detected in the NOAA-18 sample. The dashed line indicates a one-to-one relationship in residual albedo. 


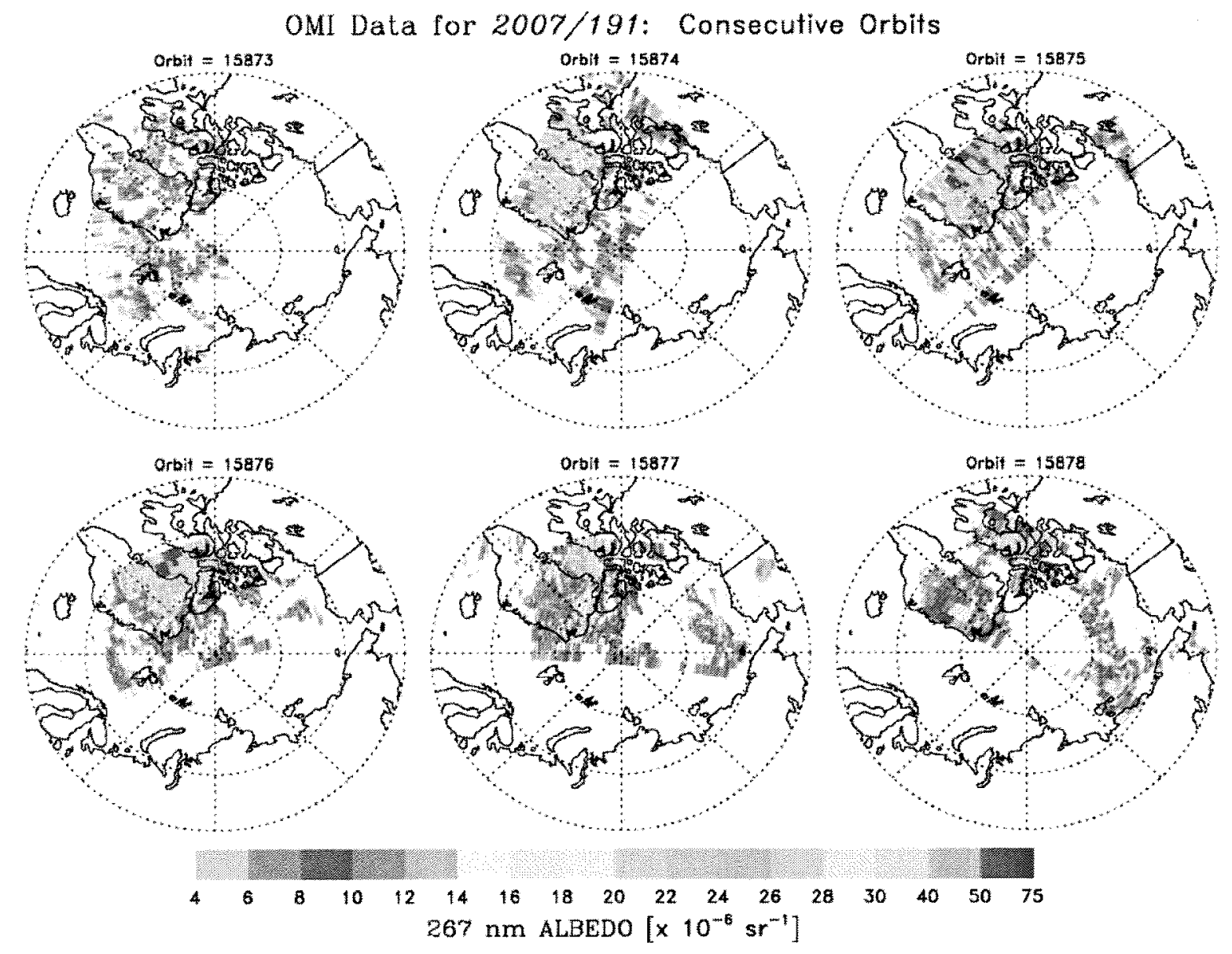

Figure 7. PMC albedo values at $267 \mathrm{~nm}$ observed by OMI for 6 consecutive orbits on 2007 day 191. Data are shown for $60^{\circ}-90^{\circ} \mathrm{N}$ latitude. Note the evolution of the bright region that is first observed at $70^{\circ} \mathrm{N}, 45^{\circ} \mathrm{E}$ in orbit 15873 . 


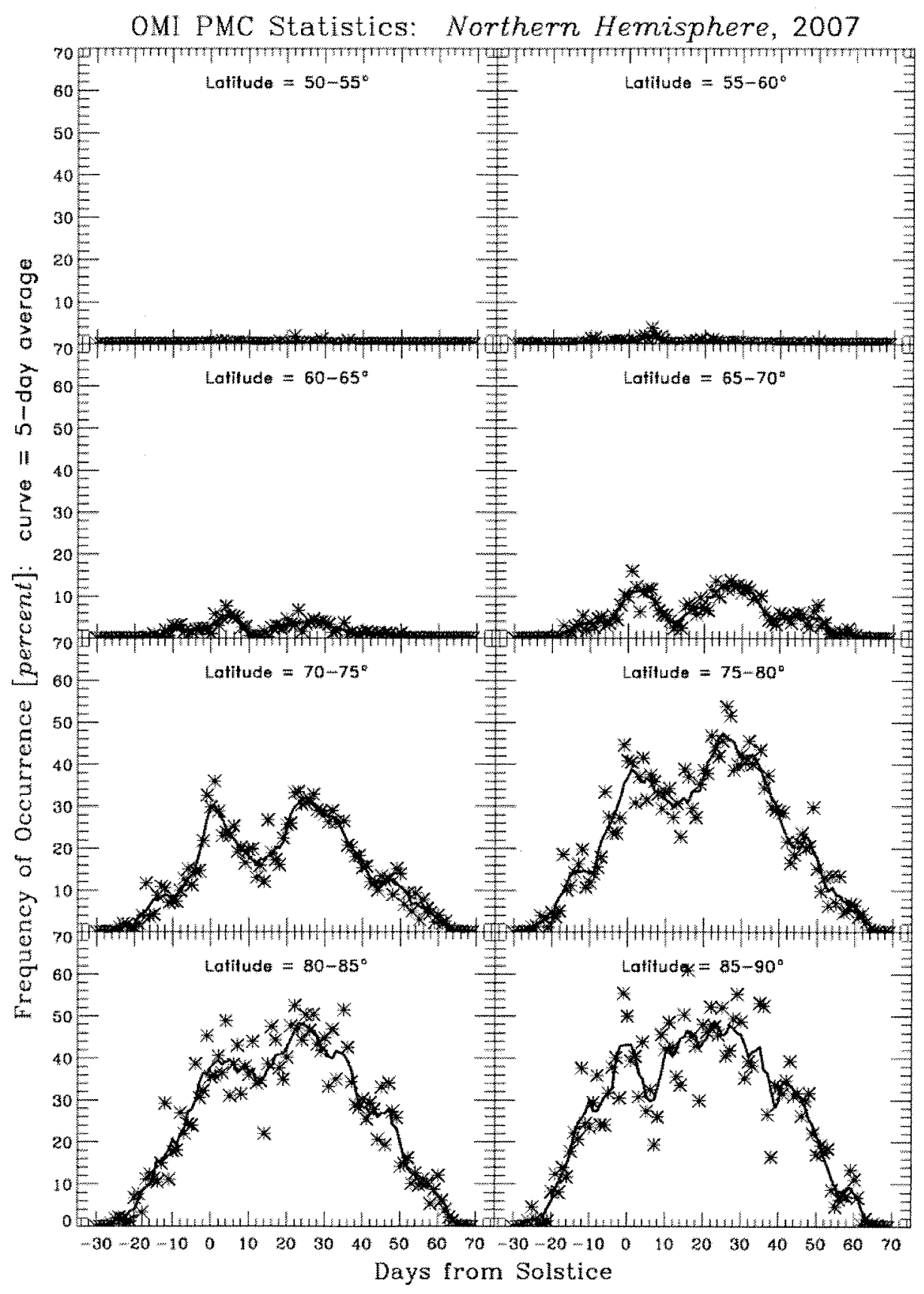

Figure 8. Daily values of OMI zonal average PMC occurrence frequency in 5 degree latitude bands between $50^{\circ}-90^{\circ} \mathrm{N}$ latitude for the Northern Hemisphere 2007 season. The solid line is a 5 -day running average. 


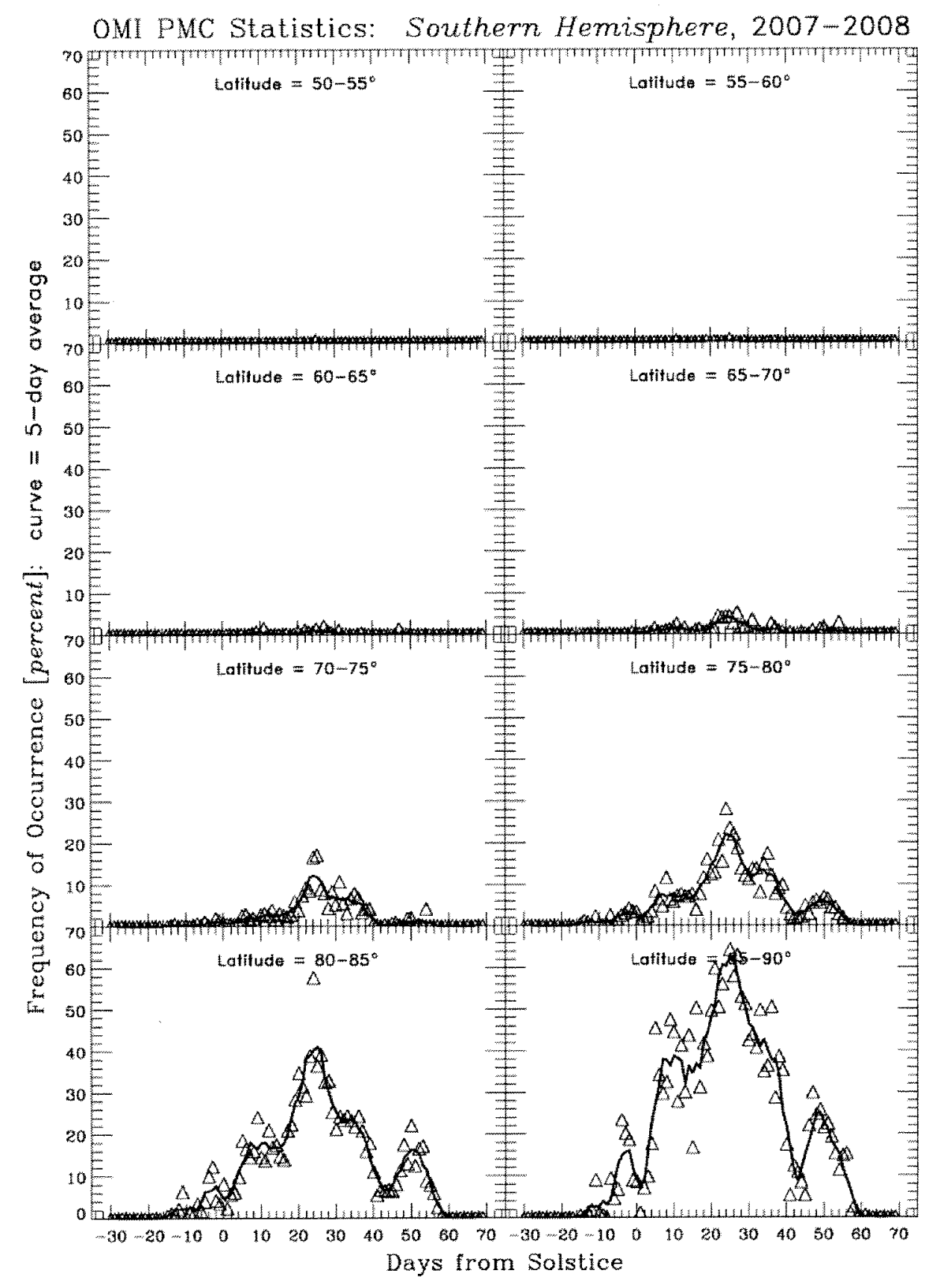

Figure 9. Daily values of OMI zonal average PMC occurrence frequency in 5 degree latitude bands between $50^{\circ}-90^{\circ} \mathrm{S}$ latitude for the Southern Hemisphere 2007-2008 season. The solid line is a 5-day running average. 